\title{
UNA APROXIMACIÓN A LOS VOCABULARIOS, GLOSARIOS Y REPERTORIOS LÉXICOS DEL ESPAÑOL EN ESTADOS UNIDOS (SIGLO XIX) ${ }^{1}$
}

\author{
AN APPROACH TO THE VOCABULARIES, GLOSSARIES AND LEXICAL \\ REPERTOIRES OF SPANISH IN THE UNITED STATES (19TH CENTURY)
}

\author{
M. ${ }^{a}$ Ángeles García Aranda \\ Universidad Complutense de Madrid \\ magaranda@filol.ucm.es
}

\begin{abstract}
RESUMEN
Este artículo tiene como objetivo presentar un panorama de la lexicografía española elaborada en Estados Unidos durante el siglo XIX. Aunque estuvo marcada, fundamentalmente, por los repertorios léxicos de Henry Neuman y Giuseppe Marco Antonio Baretti publicados en la primera mitad de la centuria y por los de Mariano Velázquez de la Cadena, no fueron los únicos diccionarios que se compusieron: junto a ellos vieron la luz otros muchos vocabularios y glosarios para acompañar gramáticas, métodos de español, manuales de composición y lectura o recopilaciones de textos literarios.
\end{abstract}

Palabras clave: lexicografía española, Estados Unidos, vocabularios, glosarios, siglo XIX.

\begin{abstract}
This paper aims to present an overview of the Spanish lexicography produced in the United States during the 19th century. Although it was marked, fundamentally, by the lexical repertoires of Henry Neuman and Giuseppe Marco Antonio Baretti published in the first half of the century and by those of Mariano Velázquez de la Cadena, they were not the only dictionaries that were composed: along with them many other vocabularies and glossaries were published to accompany grammars, Spanish methods, composition and reading manuals or compilations of literary texts.
\end{abstract}

Keywords: Spanish lexicography, United States, vocabularies, glossaries, 19th century.

\footnotetext{
${ }^{1}$ Este trabajo se enmarca dentro del Proyecto de Investigación "Biblioteca Virtual de la Filología Española. Fase IV: implementaciones y mejoras. Metabúsquedas. Gestores bibliográficos” (PID2020-112795GB-I00).
} 


\section{PUNTO DE PARTIDA: LOS PRINCIPALES DICCIONARIOS DEL ESPAÑOL EN SU HISTORIA}

Aunque la historia de los diccionarios del español está todavía por hacer, las recopilaciones sobre las principales producciones lexicográficas realizadas hasta la fecha (las incluidas, por ejemplo, en De antiguos y nuevos diccionarios del español de Manuel Alvar Ezquerra 2002, en la Lexicografía española de Medina Guerra 2003 o en Historiografía de la lingüística en el ámbito hispánico editada por Dorta, Corrales y Corbella 2007) suelen establecer, con el objetivo de conocer cómo ha evolucionado la técnica lexicográfica y cuáles son los textos referentes en la lexicografía española, una periodización que incluye etapas sobre la lexicografía medieval (glosarios, grandes repertorios medievales), sobre la lexicografía humanística (con capítulos sobre los primeros diccionarios bilingües y plurilingües del español con lenguas europeas, americanas y asiáticas), sobre el nacimiento de la lexicografía monolingüe, sobre la lexicografía extracadémica de los siglos XVIII y XIX o sobre los repertorios léxicos más importantes de los siglos XX y XXI. Entre los referentes, se suele citar a Palencia, Nebrija, Palmireno, Berlaimont, Ledel, Percyvall, Oudin, de las Casas, Franciosini, Guadix, del Rosal, Covarrubias, la Academia, Terreros, González Arnao, Núñez Taboada, Pla y Torres, Peñalver, Castro, Domínguez, editorial Gaspar y Roig, Barcia, Sociedad Literaria, Salvá, Chantreau, Valbuena, Sobrino, Cuervo, Pagés, Rodríguez Navas, editorial Larousse, editorial Vox, Casares, Moliner, Seco, Corominas y Pascual o Alvar Ezquerra, entre otros muchos.

Y entre la tipología diccionarística se suelen incluir, en función de diferentes criterios, la descripción y el contenido de los principales diccionarios etimológicos, de especialidad (náuticos, médicos, militares, de botánica), de sinónimos, ideológicos y de ideas afines, de rimas, por imágenes, de construcción o sintácticos, de uso, de dudas, de siglas, didácticos, bilingües y multilingües, de préstamos, fraseológicos, enciclopédicos o de variantes diafásicas y diastráticas. 
Aunque estos recorridos por la lexicografía del español son necesariamente limitados (lo más habitual es que se incluyan en volúmenes más amplios), suelen ceñirse a la lexicografía europea. Fuera de Europa citan, someramente y no todos, algunas producciones hispanoamericanas o asiáticas (en el contexto, normalmente, de la lingüística misionera o de la preocupación por el español que se desarrolló, especialmente a partir del XIX, en países como Argentina, Chile, México, Perú o Colombia).

Llama en este sentido la atención que apenas existan referencias a la producción lexicográfica norteamericana, concretamente a la estadounidense. Para paliar esta situación presentamos a continuación un panorama, descriptivo y necesariamente limitado, de los repertorios léxicos con el español y el inglés en Estados Unidos durante el siglo XIX, centuria especialmente prolífica en lo que a publicación de textos lexicográficos se refiere y desatendida por la investigación metalexicográfica.

\section{EL ESPAÑOL EN ESTADOS UNIIDOS}

Aunque la enseñanza del español en la América del norte tuvo cierta importancia desde mediados del siglo XVII, debido al intercambio comercial con las colonias americanas, es en el siglo XIX cuando se publican obras gramaticales y lexicográficas con diferentes objetivos, cuando se publican los primeros periódicos en nuestra lengua (El Habanero, El Mensajero Semanal, El Redactor, Mercurio de Nueva York, El Noticioso de Ambos Mundos, La Verdad...) y cuando entra con fuerza en los planes de estudio de las universidades americanas gracias a las clases que, en muchos casos, impartían emigrados españoles e hispanoamericanos ${ }^{2}$.

En esta centuria se siente el español como una lengua necesaria e importante en los Estados Unidos que debe ser aprendida por quienes allí viven: la cercanía

\footnotetext{
${ }^{2}$ Para la historia de la enseñanza del español en Estados Unidos, véanse Grattan Doyle 1926, Leavitt 1961, Nichols 1945, Spell 1927, Sánchez Pérez 1992 y, especialmente, Vilar García 1996 у 2008.
} 
con Hispanoamérica, las relaciones comerciales o la literatura española son algunas de las razones que motivan este interés. Ello acarrea la presencia de cátedras de lengua y literatura españolas o de lenguas modernas en la City University of New York, en el Saint Mary's College de Baltimore, en la Universidad de Harvard, en la Universidad de Chicago, en la Universidad de Yale, en la Universidad de Filadelfia, en la Universidad de Maryland, en la Universidad de Princeton o en el King's College de Nueva York, en donde prestigiosos profesores de español (de origen peninsular e hispanoamericano que emigrados por razones políticas y sociales y, aunque con otras formaciones, se dedicaron a la enseñanza del español y de otras lenguas modernas) desarrollan su labor docente y elaboran materiales para sus clases que, con frecuencia, dieron a la imprenta.

Es así como en esos años ven la luz en Nueva York, Filadelfia, Baltimore o Boston las gramáticas, las ortografías, los diccionarios y los diálogos, muchas veces adaptaciones de conocidos métodos de enseñanza (Dufief, Ollendorff, Robertson, Seidenstücker, Ahn, Pestalozzi o Berlitz), de Mariano Velázquez de la Cadena, de Mariano Cubí y Soler, de Julio Soler, de Francisco Javier Vingut, de Francis Salas, de Thomas S. Brady, de Emanuel del Mar, de José J. Villarino, de Henry Neuman y Giuseppe Marco Antonio Baretti, de Alexander H. Monteith, de Joseph Salkeld, de Ramón Palenzuela y Juan de la C. Carreño, de Maximilian Schele de Vere, de Norman Pinney y Juan Barceló, de Louis Ernst o de Alberto de Tornos, así como de otros textos más breves y encaminados exclusivamente a mostrar a los usuarios los rudimentos para desenvolverse en sus estancias en España o en Hispanoamérica, obras que, en algunos casos, conocieron un gran número de reediciones y reimpresiones (cf. Esparza \& Niederehe, 2012 y 2015; Vilar García 2008).

El público al que van dirigidas estas obras también es heterogéneo: algunas están destinadas a estudiantes universitarios, otras se compusieron para alumnos no universitarios, otras pretenden acercar el español al público general, otras tienen fines comerciales y otras tratan de satisfacer las necesidades de un público específico. 
Por otro lado, los prólogos y las páginas preliminares coinciden en señalar la importancia de aprender español, las bondades de los diferentes métodos en el aprendizaje de español o el compromiso de enseñar nuestra lengua en poco tiempo. También es frecuente que los autores de los textos comenten las fuentes o series preparatorias o retrospectivas que motivaron sus publicaciones. Son recurrentes la alusión a la Academia Española, referente entre la mayor parte de los profesores de español ${ }^{3}$, y la mención a "los mejores autores", quienes reciben las alabanzas o el reconocimiento como inspiradores de las obras.

Así mismo, parece evidente que los autores debían conocer la tradición europea, pues, además de que hasta comienzos del siglo XIX los textos para la enseñanza del español se importaban de Londres, algunos de ellos conocieron ediciones inglesas y americanas y otros no son sino versiones o adaptaciones de obras publicadas en Inglaterra ${ }^{4}$.

\footnotetext{
${ }^{3}$ La presencia de la Academia Española en Estados Unidos se aprecia, además de en las citas que realizan los autores de los manuales, en textos como los Elementos de la lengua castellana fundados en los principios establecidos por la Academia española y en el uso de los autores clásicos de Mariano Velázquez de la Cadena (New York, G. Gratton, 1810), obra reeditada en varias ocasiones más (1824, 1827, 1833 y 1845 en Guatemala) que compendia la ortografía, la etimología, la sintaxis y la prosodia académicas. Según la Sociedad Patriótica de La Habana se trata de una "de las mejores en su clase, siendo recomendable por su brevedad, claridad y precisión", por lo que "la creemos preferible a otra cualquiera en nuestras escuelas de primeras letras. Desearíamos por lo mismo que la Sección [de Educación] se sirviese recomendarla á los maestros para simplificar el estudio de nuestra lengua" (1824, 2. ${ }^{\mathrm{a}}$ ed.: 3 ). ${ }^{4}$ Nos referimos a los textos de Richard Percyvall (Dictionaire in spanish, english and latine, Londres, John Jackson, 1591), John Minsehu (A Spanish gramar, Londres, Edmund Bollifant, 1599 y A dictionary in Spanish and English, Londres, John Haviland, 1623), James Howell (Lexicon tetraflotton, Londres, Samuel Thomson, 1660 y A new English grammar, Londres, T. Williams, H. Brome y H. Marsh, 1662), John Stevens (A new spanish gramar y A Vocabulary, Londres, T. Meighan, J. Batley y T. Cox, 1725), Pedro Pineda (Corta y compendiosa arte para aprender a hablar, leer y escrivir la lengua española, Londres, T. Woodward, 1726; Nuevo diccionario, español e inglés e inglés y español, Londres, F. Gyles-T. Woodward-T. Cox y J. Clarke-A. Millar-P. Vailland, 1740 y A short and easy introduction to the rudiments of the Spanish tongue, Londres, Pedro Pineda, 1750), Hipólito San José Giral Delpino (A New Spanish and English dictionary, Londres, A. Millar, J. Nourse and P. Vaillant, 1763 y A new spanish grammar, Londres, J. Nourse, 1766), Raimundo del Pueyo (A new Spanish grammar, Londres, F. Wingrave, 1792) o Thomas Connelly y Tomás Higgins (Diccionario nuevo y complete de las lenguas española e inglesa, inglesa y española, Madrid, Imprenta Real, 1797).
} 


\section{LOS REPERTORIOS LÉXICOS CON EL ESPAÑOL Y EL INGLÉS EN ESTADOS UNIDOS}

El corpus que ahora se presenta se ha realizado a partir, fundamentalmente, de la información contenida en tres fuentes principales: el volumen de Mar Vilar García dedicado al español en EE.UU. (2008), la BICRES (Bibliografía cronológica de la lingüística, la gramática y la lexicografía del español, 2012 y 2015) y la Biblioteca Virtual de la Filología Española (www.bvfe. es), recopilaciones que atesoran un número considerable de ediciones y reimpresiones que, además en el caso de la BVFE, pueden consultarse libremente.

Dado que todos son repertorios bilingües (número de lenguas) redactados en el siglo XIX (eje temporal) y teniendo en cuenta que todos se compusieron con fines didácticos o pedagógicos, hemos utilizado para establecer su tipología los siguientes criterios: extensión de las entradas (grandes diccionarios vs. repertorios manuales, abreviados y turísticos) y ordenación de los materiales (alfabéticos vs. temáticos).

\subsection{GRANDES Diccionarios alFabéticos}

Entre los grandes diccionarios alfabéticos publicados durante el siglo XIX en suelo estadounidense destacan, sin duda alguna, dos: el de Neuman y Baretti por un lado y el de Velázquez de la Cadena, por otro.

\subsubsection{Henry Neuman y Giuseppe Marco Antonio Baretti ${ }^{5}$}

Henry Neuman, lexicógrafo de origen británico, desarrolló una importante labor adaptando y ampliando diccionarios bilingües del español y del inglés para la editorial Vernor and Hood. En 1799 firma A marine pocket-dictionary, of the Italian, Spanish, Portuguese and German languages; en 1802 se encarga de la revisión y ampliación del Diccionario nuevo de las lenguas

\footnotetext{
5 Para conocer con detalle la vida y la obra completa de estos autores, véanse Vilar García (2008) y las fichas biobibliográficas que Estela Calero ha realizado para la Biblioteca Virtual de la Filología Española (www.bvfe.es).
} 
española e inglesa elaborado por Thomas Connelly y Thomas Higgins, y, desde 1823, trabaja, junto a Giuseppe Marco Antonio Baretti (lexicógrafo italiano afincado en Londres), en la publicación de $A$ pocket dictionary of the Spanish and English languages (que también se publicó en Filadelfia) y de Dictionary of the Spanish and English languages (1827), compilación que fue conocida mundialmente, por el gran número de ediciones europeas y americanas, como Neuman \& Baretti's Dictionary (cfr. Hartmann, 2003; Martín-Gamero, 1961; Mühlschlegel, 2011; Piccioni, 1899; Steiner 1970).

En Estados Unidos, el Neuman E Baretti's Dictionary se reimprimió anualmente en las décadas de los treinta, los cuarenta y los cincuenta, en Boston y Nueva York a partir de la 4 . $^{\mathrm{a}}$ edición londinense, 1827, lo que lo convirtió en el diccionario más importante del español en tierras americanas (con elogios y críticas).

El nuevo repertorio tiene como objetivo recoger "not only many thousand more words than are to be found in the most copious vocabularies, but also much practical and useful information, correct data from which the nature and operations of the human mind in Spain and in England may be deduced; more facts, principles and terms, now used in the sciences, arts, manufactures, and commerce, than in any similar dictionary hitherto published, and likewise more of the modem words in the polite or familiar conversation of both countries" (1827, iv).

El Dictionary de Neuman y Baretti consta de dos extensos volúmenes (el primero español-inglés y el segundo inglés-español), con variados artículos lexicográficos ordenados alfabéticamente en los que se da cuenta de información gramatical (incluida la fónica) y semántica. La microestructura suele ser muy sencilla: entrada/lema, información gramatical abreviada, marcas diatécnicas, acepciones (en ocasiones sinonímicas o limitadas a la equivalencia en la otra lengua y, las más de las veces, con definiciones y explicaciones detalladas) y unidades pluriverbales (a veces, con marcas de transición semántica). Informaciones que pueden verse en las Tablas 1 y 2 : 


\begin{tabular}{|c|c|}
\hline 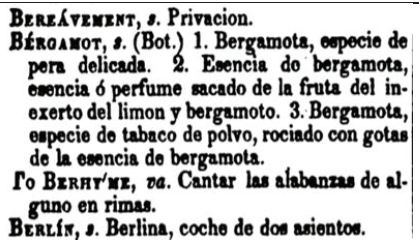 & 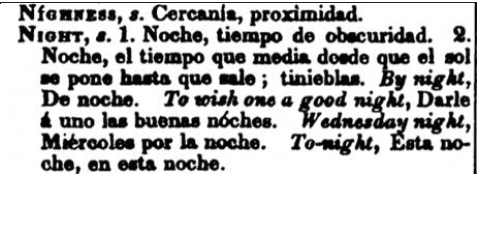 \\
\hline 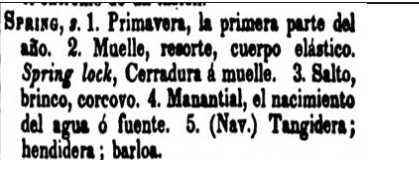 & 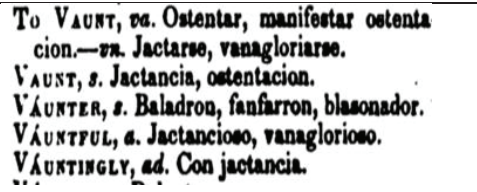 \\
\hline
\end{tabular}

TABla 1. Muestra del Neuman \& Baretti's Dictionary (1827), parte inglés-español .

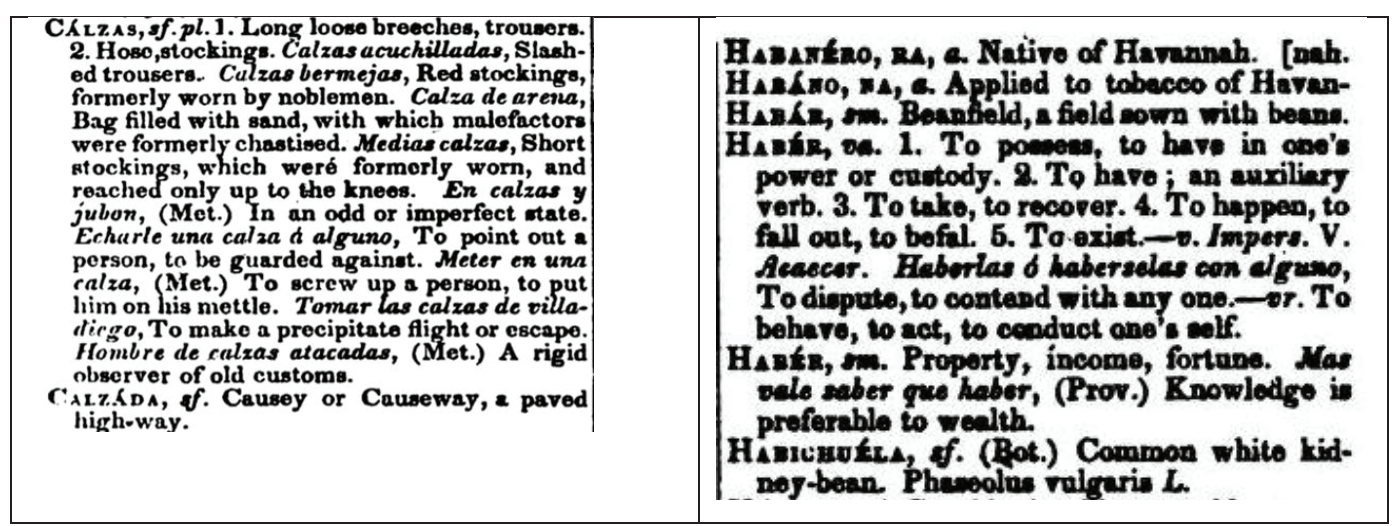

TABLA 2. Muestra del Neuman \& Baretti's Dictionary (1827), parte español-inglés ${ }^{7}$.

El diccionario de bolsillo o A pocket dictionary of the Spanish and English languages (Filadelfia, H. C. Carey \& I. Lea, 1826) es también bidireccional, y no es, pese a su extensión (un volumen de más de setecientas páginas), sino una recopilación simplificada del diccionario anterior, tal y como se aprecia en los ejemplos recogidos en la Tabla 3.

\footnotetext{
${ }^{6}$ Neuman y Baretti: Dictionary of the Spanish and English languages (Boston, 1827). http:// archive.org/details/neumanandbarett04baregoog?view=theater.

7 Neuman y Baretti: Dictionary of the Spanish and English languages (Boston, 1827). https://books.google.es/books?id=fgYtAAAAYAAJ\&printsec=frontcover\&dq=baretti+dictionary\&lr=\&as_brr=1\&source=gbs_book_other_versions_r\&hl=es ${ }^{*} \mathrm{~V}=$ onepage $\& q=b a r e-$ tti\%20dictionary\&f=false.
} 


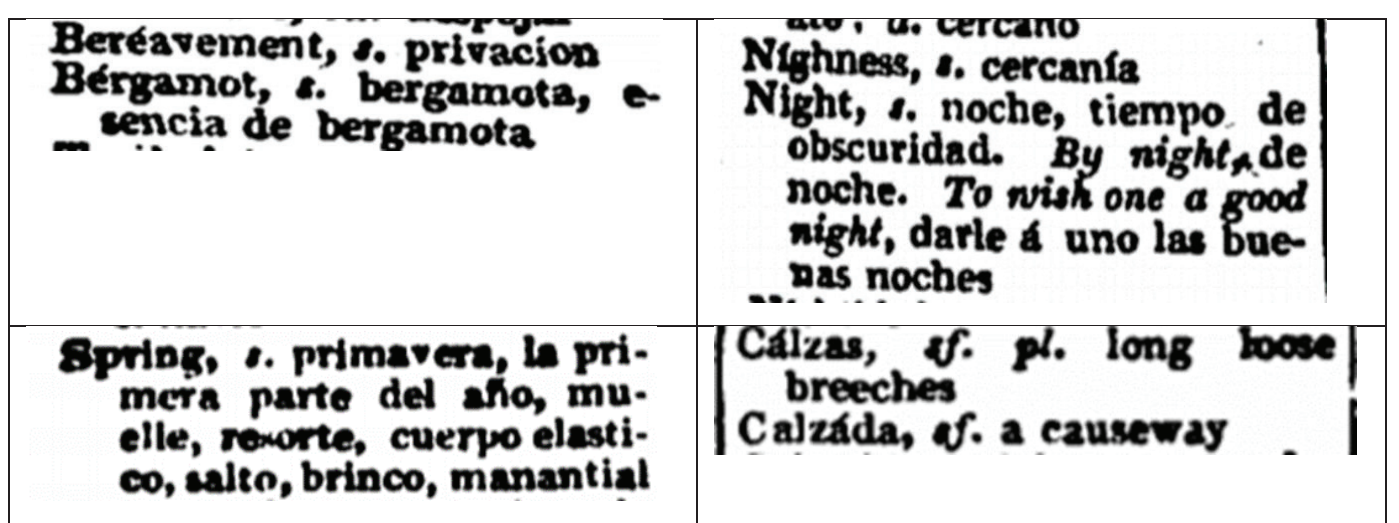

TABLA 3. Muestra de A pocket dictionary of the Spanish and English languages $(1826)^{8}$

El repertorio de Newman y Baretti fue corregido y reformado en 1831 por Mateo Seoane bajo el título de Diccionario de las lenguas española e inglesa de Newman y Baretti (Londres) y, en las ediciones posteriores, Diccionario de las lenguas española e inglesa de Neuman y Baretti, en el cual se definen todas las palabras en sus diversas significaciones. Aumentada con un gran número de palabras sacadas de los mejores autores tanto ingleses como españoles (Londres, 1854). Esta nueva versión fue el punto de partida para la adaptación que realizó Mariano Velázquez de la Cadena en Estados Unidos: Seoanes Neuman and Baretti. Abridged by Velazquez. A dictionary of the Spanish and English languages. Abridged from the author's larger work (D. Appleton and Company, Nueva York-Cincinnati, 1858).

\subsubsection{Mariano Velázquez de la Cadena}

Mariano Velázquez de la Cadena, mexicano de nacimiento, es, seguramente, una de las figuras más importantes en la enseñanza del español en Norteamérica en el siglo XIX. Tras una intensa actividad política en España y ante la situación que vivía su país natal, decidió instalarse en Nueva York y dedicarse a impartir clases de lengua y literatura y a componer nuevos métodos

\footnotetext{
${ }^{8}$ Neuman y Baretti: A pocket dictionary of the Spanish and English languages (Filadelfia, 1826). https://books.google.es/books?id=YCQSAAAAIAAJ\&printsec=frontcover ${ }^{\mathrm{V}} \mathrm{V}=$ onepage\&$q \& f=$ false.
} 
de enseñanza para facilitar el aprendizaje a sus alumnos. Fue catedrático de Lengua y Literatura españolas en el Columbia College, autor de diversas obras sobre enseñanza del español (solo o en compañía de otros colegas) y adaptador del método Dufief y del método Ollendorff de enseñanza de lenguas (cfr. Gallardo Barbarroja, 2002; Garriga \& Gallego, 2008; Sánchez Pérez, 1992; Vilar García 2008).

En sus textos es constante la preocupación por la renovación metodológica en la enseñanza de español y el deseo por proporcionar a sus estudiantes nuevos materiales que puedan aumentar y mejorar su competencia comunicativa.

Entre sus obras lexicográficas destacan Seoanes Neuman and Baretti. Abridged by Velazquez. A dictionary of the Spanish and English languages (Nueva York-Cincinnati, D. Appleton and Company, 1858), adaptación de Seoane, y el que será, seguramente, uno de los diccionarios más importantes de la centuria: A pronouncing dictionary of the Spanish and English languages (D. Appleton and Company, Nueva York, 1852), repertorio que con el paso del tiempo se publicó como Diccionario de pronunciación de las lenguas inglesa y española.

El primero de ellos es, como se ha dicho, una adaptación del Newman y Baretti a partir de la versión de Seoane, poco original, pues (véase la Tabla 4).

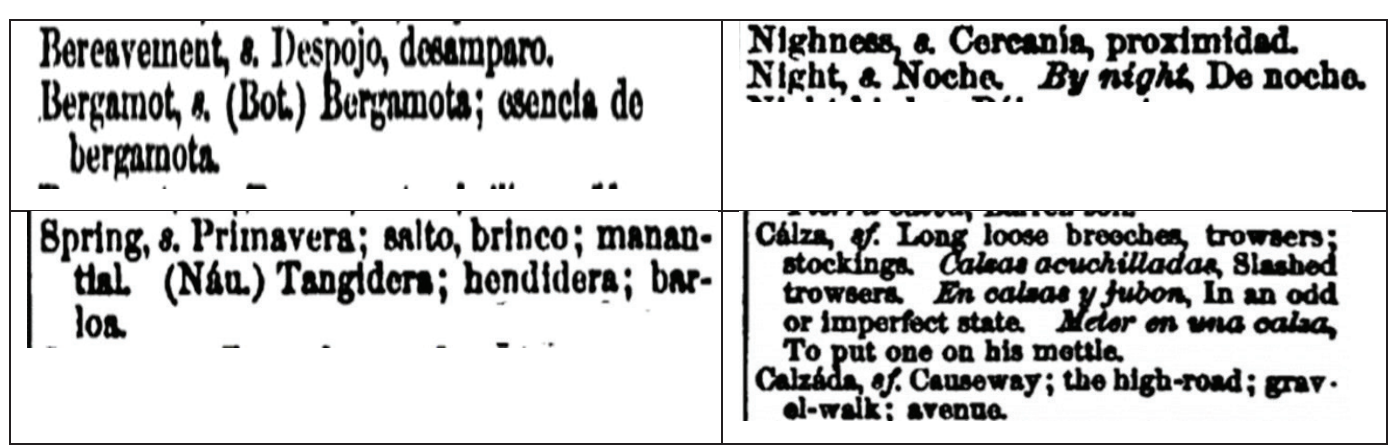

TABla 4. Muestra de A dictionary of the Spanish and English languages (1858)9

9 Velázquez de la Cadena: A dictionary of the Spanish and English languages (1858). https://archive.org/details/adictionaryspan01cadegoog?view=theater. 
Los diccionarios de pronunciación de Velázquez de la Cadena (Garriga \& Gallego, 2008) se compusieron a partir de diversas fuentes que el propio autor reconoce en la portada y en las páginas preliminares: Seoane, Neuman y Baretti, la Academia Española, Salvá, Terreros, las Etimologías Castellanas de Cabrera, las Voces Cubanas de Pichardo, Webster, el Diccionario Worcester y el tratado Americanisms de Bartlett.

El volumen español/inglés contiene algo más de 42500 entradas, mientras que el inglés/español rebasa las 49000 entradas. Velázquez de la Cadena recogió para su diccionario, como había hecho antes y tal y como también habían hecho sus fuentes, voces técnicas y voces dialectales que aparecen en artículos lexicográficos compuestos por lema/entrada, información gramatical, acepciones (definiciones lexicográficas de distinto tipo) y unidades pluriverbales (subentradas). La novedad del repertorio es la inclusión, tras la entrada, de pronunciaciones figuradas, lo que respondía al interés, cada vez mayor en la enseñanza de lenguas, en Europa y en América, por el componente oral de la lengua. Los ejemplos recogidos en la Tabla 5 son buena muestra de ello.

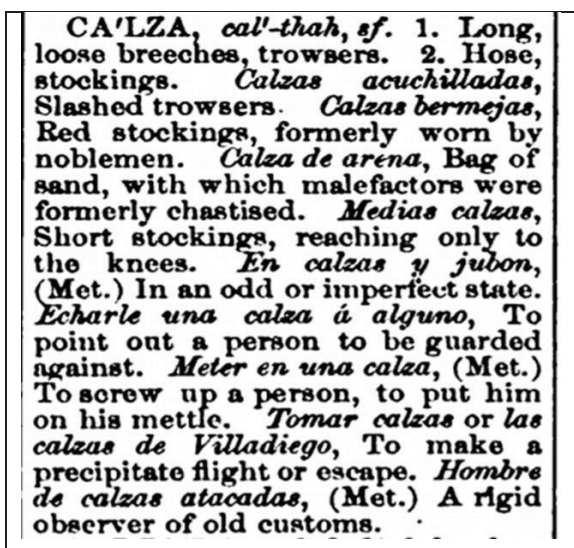

SI'LLA, seel'-lyah, of. 1. Chair, movable sent. 2. See, the reat of episcopal power; the diocese of a bishop. 8. Silla or silla do muntar, Saddle. 4. Seat. 5. Seat, anus. Sillit de manas, Sedan-chair. Silla pot trona, Arun or elbow-chair; a lazy chair. Silla de posta, Post-chaise. Silla volante, A lightit gig. Silla de rejilla or de junoo, Cane or baunboobottomed chair. Silla de palo, Wooden-bottorned chair. Sitha do columpio, Rocking-chair. Sillas do caoba con asiento do crin or clin, $\mathrm{Ma}$ hogany hair-bottomed chairs. Sillas girantes, Plvot chairs. De silla á sille, Face to fuce. Hombre do ámbus sillus or de tadas sillas, A man of general information; a clever fellow.

TABla 5. Muestra de A pronouncing dictionary of the Spanish and English languages (1852) ${ }^{10}$

${ }^{10}$ Velázquez de la Cadena: Velázquez de la Cadena: A pronouncing dictionary of the Spanish and English languages (1852). https://books.google.es/books?id=KCsaAQAAMAAJ\&print$\underline{\mathrm{sec}}=$ frontcover\&hl$=\mathrm{es} \&$ source $=\mathrm{gbs}$ ge summary $\mathrm{r} \& \mathrm{cad}=0^{\#} \mathrm{v}=$ onepage $\& \mathrm{q} \& \mathrm{f}=\mathrm{false}$. 
La obra tuvo éxito y alcanzó una gran difusión gracias a sus numerosas ediciones (1852, 1854, 1856, 1860, 1861, 1863, 1875, 1899... hasta la actualidad), razón por la que la Academic Learning Company (California, EE. UU.), compró los derechos de publicación de la obra en 2003 y lanzó al mercado la división Velazquez Press.

\subsection{Vocabularios en manuales de enseñanza}

Junto a los grandes diccionarios alfabéticos, se compusieron otros muchos vocabularios y glosarios para acompañar métodos de español, gramáticas, manuales de composición y lectura o recopilaciones de textos literarios.

\subsubsection{Vocabularios escondidos en gramáticas}

Parte de la lexicografía bilingüe hispanoinglesa estadounidense se publicó junto a gramáticas o textos gramaticales, que solían incluir listas de palabras bidireccionales que solo recogen léxico básico y su traducción o equivalencia en la otra lengua en sencillos artículos lexicográficos con el fin de ayudar en el proceso de aprendizaje. Es el caso, por ejemplo, de The principles of grammar, being a compendious treatise on the languages, English, Latin, Greek, German, Spanish and French de Solomon Barrett (King \& Baird, Filadelfia, 1852, reeditada en Boston, Geo. C. Rand y Avery, Boston, 1861), del Practical course with the Spanish language de Herman Monsanto y Louis Languellier (Nueva York y Chicago, 1875); véase una muestra en la Tabla 6, de A grammar of the modern Spanish language as now written and spoken in the capital of Spain de William Ireland Knapp (Boston, Ginn \& Co., Boston, 1882, reeditada en 1890 y 1900) o de A brief Spanish grammar with historical introductions and exercises de August Hialmar Edgren (Boston, D. C. Heath \& Co., 1891, reeditada en 1899, 1913). 


\begin{tabular}{|c|c|c|}
\hline $\begin{array}{l}\text { L. } \\
\text { labrador, m. farmer. } \\
\text { lado, m. side. } \\
\text { ladron, m. thief. } \\
\text { lágrima, f. tear. } \\
\text { lámpara, f. lamp. } \\
\text { largo, long. } \\
\text { Latin, Latin. } \\
\text { lavandera, f. vasher- } \\
\text { woman. } \\
\text { lápiz, m. pencil. } \\
\text { lavar, 1. to vash. } \\
\text { leccion, f. lesson. } \\
\text { leche, f. mill. } \\
\text { leer, 2. to read. } \\
\text { legua, f. league. } \\
\text { legumbre, f. vegetable. } \\
\text { leido, reud. } \\
\text { léjos, far. } \\
\text { lengua, f. language. }\end{array}$ & $\begin{array}{l}\text { lugar, m. place, spot. } \\
\text { Luisa, Louisa. } \\
\text { luna, f. moon. } \\
\text { luz, f. light. } \\
\qquad \text { LL. } \\
\text { llamar, 1. to call. } \\
\text { llamarse, 1. to be called. } \\
\text { llave, f. key. } \\
\text { llega, arrives. } \\
\text { llegar, 1. to arrive. } \\
\text { llegado, arrived. } \\
\text { lleno, full. } \\
\text { llevar, 1. to take. } \\
\text { llora, veeps. } \\
\text { llorar, 1. to cry, to veep. } \\
\text { llover, 2. to rain. } \\
\text { lluvia, f. rain. }\end{array}$ & $\begin{array}{l}\text { marcharse, 1. to depart. } \\
\text { marido, m. hasband. } \\
\text { marinero, m. sailor. } \\
\text { mas, more. } \\
\text { máscara, f. mask. } \\
\text { matar, 1. to kill. } \\
\text { máxima, f. maxim. } \\
\text { la mayor parte, the most. } \\
\text { media, f. stocking. } \\
\text { médico, m. physician. } \\
\text { medio, m. means, way. } \\
\text { medir, } 3 . \text { to measure. } \\
\text { memoria, f. memory. } \\
\text { menester, necessary. } \\
\text { mentira, f. falschood. } \\
\text { mercader, m. merchant. } \\
\text { mercancias, f. pl. goods. } \\
\text { merecer, 2. to deserve. } \\
\text { mérito, m. merit. } \\
\text { mes, m. month. } \\
\text { mesa, f. table. }\end{array}$ \\
\hline
\end{tabular}

\begin{tabular}{|c|c|c|}
\hline $\begin{array}{l}\text { obstinate, porfiado. } \\
\text { to obtain, obtener, } 2 \text {. } \\
\text { conseguir, } 3 . \\
\text { occasion, ocasion, f. } \\
\text { to oceupy, ocupar, } 1 \text {. } \\
\text { of, de. } \\
\text { to offind, ofender, } 2 \text {. } \\
\text { to offer, ofreoor, 2. su- } \\
\text { ministrar, 1. } \\
\text { officer, oficial, m. } \\
\text { office, escritorio, m. } \\
\text { often, a menudo. } \\
\text { oil, acoite, m. } \\
\text { old, viejo. } \\
\text { on, sobre. } \\
\text { only, solamente. } \\
\text { to open, abrir, } 3 \text {. } \\
\text { opened, abierto. } \\
\text { opinion, opinion, f. con- } \\
\text { copto, m. } \\
\text { orange, naranja, f. }\end{array}$ & $\begin{array}{l}\text { painting, pintura, } \mathrm{f} \text {. } \\
\text { palace, palacio, m. } \\
\text { paper, papel, m. } \\
\text { parents, padres, m. pl. } \\
\text { park, parque, m. } \\
\text { part, parte, } \mathrm{f} . \\
\text { to pass, pasar, } 1 . \\
\text { past, pasado. } \\
\text { paternal, paternal. } \\
\text { patience, paciencia, f. } \\
\text { patient (sick person), } \\
\text { enfermo, m. } \\
\text { to pay, pagar, } 1 . \\
\text { peace, paz, } \mathrm{f} \text {. } \\
\text { pear, pera, f. } \\
\text { to peol, desciscarar, } 1 . \\
\text { pencil, lapiz, m. } \\
\text { penlmifo, cortaplumas, } \\
\text { m. } \\
\text { people, gente, f. } \\
\text { to people, poblar, } 1 .\end{array}$ & $\begin{array}{l}\text { polite, cortis, comedido. } \\
\text { Pompey, Pompeyo. } \\
\text { poor, pobre. } \\
\text { port, puerto, m. } \\
\text { possession, pasesion, f. } \\
\text { possible, posible. } \\
\text { post, correo, m. } \\
\text { posterity, posteritad, } \mathrm{f} . \\
\text { postman, cartero, m. } \\
\text { post-office, correo, m. } \\
\text { pound, libra, f. } \\
\text { power, poder, m. } \\
\text { powerful, poderoso. } \\
\text { to practise, practicar, } 1 . \\
\text { praise, alabanza, } \mathrm{f} . \\
\text { to praise, alabar, } 1 . \\
\text { precept, prccepto, m. } \\
\text { to prefer, preferir, } 3 . \\
\text { to prepare, preparar, } 1 . \\
\text { prerogative, preroga- } \\
\text { tiva, f. }\end{array}$ \\
\hline
\end{tabular}

TABla 6. Muestra de Practical course with the Spanish language de Herman Monsanto y Louis Languellier $(1875)^{11}$

\subsubsection{Vocabularios en manuales de composición y lectura}

Los libros de composición escrita y lectura también incorporan con frecuencia, para facilitar la tarea al estudiante, vocabularios o repertorios léxicos con las voces aparecidas en los textos y escritos que compendian. Suelen ser, tanto macroestructural como microestructuralmente, sencillas compilaciones que dan cuenta de las equivalencias léxicas entre las dos lenguas.

\footnotetext{
${ }^{11}$ Monsanto y Languellier: Practical course with the Spanish language (1875). https://archive. org/details/combinedspanishm00tornrich?view=theater.
} 
Una muestra de ellos son los "vocabularies" que se encuentran al final de The Spanish translator de Mariano Cubí y Soler (Baltimore, Fielding Lucas, jr., ca. 1841), de A new Spanish reader consisting of passages from the most approved authors, in prose and verse, arranged in progressive order, for the use of those who wish to obtain easily a practical knowledge of the Castilian language de Mariano Velázquez de la Cadena (Nueva York-Filadelfia, D. Appleton \& Co.-Geo. S. Appleton, 1849), de Modern Spanish readings embracing text, notes, and an etymological vocabulary de William Ireland Knapp (Boston, Ginn \& Company, 1887), de First Spanish readings de John E. Matzke (Boston, 1899); véase la Tabla 7, o de De Exercises in spanish composition for first and second year classes de Jeremiah Denis Matthias Ford (Boston-Nueva York-Chicago, D. C. Heath \& Co., 1899).

\begin{tabular}{|c|c|}
\hline 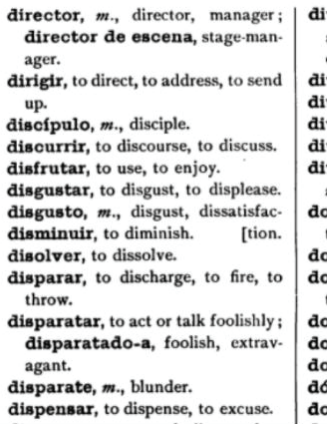 & 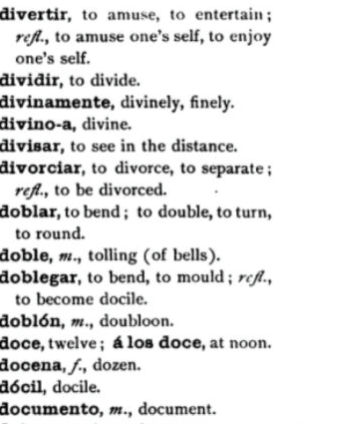 \\
\hline 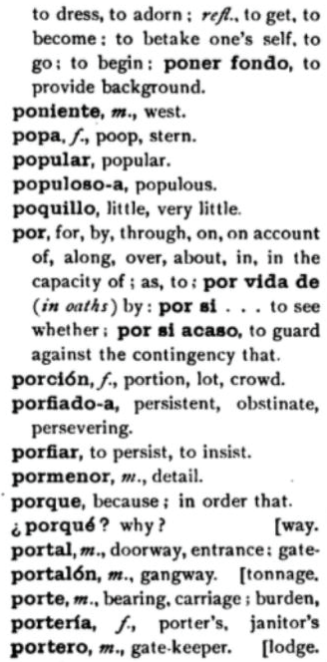 & 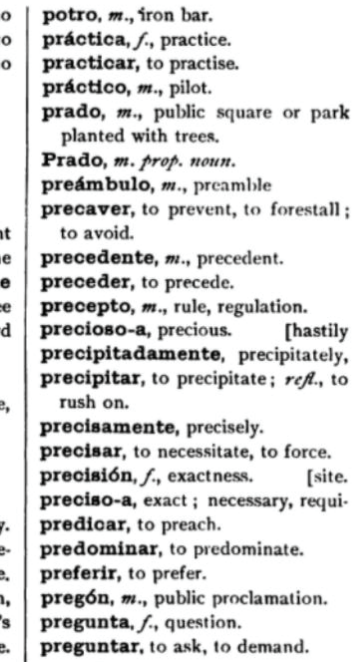 \\
\hline
\end{tabular}

TABla 7. Muestra de First Spanish readings de John E. Matzke (1899) ${ }^{12}$

12 Matzke: First Spanish readings (1899). https://babel.hathitrust.org/cgi/pt?id=uc1.32106 $\underline{009019602 \& \text { view=1up\&seq }=5 .}$. 


\subsection{REPERTORIOS DE ESPECIALIDAD}

Finalmente, el desarrollo de la lexicografía de especialidad que se produce en esta centuria también tiene representantes al otro lado del Atlántico. El progreso y los avances que trajo consigo el siglo XIX impulsaron la entrada de léxico de especialidad en los diccionarios generales y la publicación de numerosos repertorios sobre muy diversas temáticas científicas y técnicas, tanto monolingües como bilingües (García Aranda, 2012 ${ }^{13}$ ).

Como señaló Manuel Alvar Ezquerra $(1992,14)$ "los diccionarios son obras de carácter didáctico que, por ello, deben atender las necesidades de los usuarios", y en esta centuria los lectores, es claro, necesitaban diccionarios especializados en varias lenguas para resolver los problemas que generaban, por un lado, las voces terminológicas y, por otro, el desconocimiento de lenguas extranjeras. Para ellos se componen, entre otros, el Diccionario tecnológico inglés-español y español-inglés de los términos y frases usados en las ciencias aplicadas, artes industriales, bellas artes, mecánica, maquinaria, minas, metalurgia, agricultura, comercio, navegación, manufacturas, arquitectura de Néstor Ponce de León (Nueva York, N. Ponce de León, 1893); véanse los ejemplos de las Tablas 8 y 9, o el Mercantile dictionary de I. de Veitelle (Nueva York, D. Appleton and Company, 1864).

\footnotetext{
${ }^{13}$ En este trabajo se citan textos de léxico marítimo (como el Diccionario marino español-inglés para el uso del Colegio Naval de Juan José Martínez de Espinosa y Tacón, 1849, el Diccionario de los términos y frases de marina, español-francés-inglés: obra útil para las marinas militar y mercante de Antonio Ferry y Rivas, 1899), de léxico militar (como el Diccionario español-alemán que contiene las palabras más necesarias para el uso de los soldados españoles en Alemania, 1807, el Vocabulario francés-español de términos de artillería de P. de la Llave, 1846, el Vocabulario militar francés, inglés, español de Joaquín M. Enrile, 1853, el Diccionario militar, etimológico, histórico, tecnológico, con dos vocabularios francés-español y alemán-español de José Almirante, 1869, o el Diccionario militar: con un vocabulario español-francés-alemán de Nicolás Estévanez, 1897), de léxico tecnológico (como el Diccionario tecnológico inglés-español, Antonio Cañada y Gisbert, 1878), de léxico comercial (como El comerciante español [...] con fraseología mercantil en español y alemán de J. E. Gómez Mier, 1854, la Nueva correspondencia comercial francesa-español, que contiene la definición y explicación del lenguage comercial y económico de José M. Lopes, 1864, o el Nuevo diccionario francés-español y español-francés. Comercial de Adolfo M. Rumeau, 1868) o de léxico religioso (como el Glossaire français-espagnol de termes religieux de Juan Bautista Vanriest, 1898).
} 
caterer, proveedor, abastecedor || fondista, hos telero que lleva comidas á los festines, \&c caterpillar (a.) oruga.

catharine ziheel (arq.) rosa, gran ventana circular, cerrada por lo comun con vidrios de colores (pir) rueda de fuegos artificiales.

cathartic, purgante, catártico.

cathedral (arq.) catedral.

catheter (cir.) algalia, sonda, bujía.

cathetometer (arm.) catetómetro.

cathetus (geo.) cateto (mec.) cateto, eje que pasa por el centro de un cuerpo cilindrico (arq.) cateto, perpendicular que pasa por el centro de la voluta del capitel jónico.

cathode (elec.) polo negativo.

catholicon (farm.) catolicon, panacea univer-

sal, sánalo-todo. préscription (farm. $)$ prescripcion, receta. presence (q.) presencia.

present, presente (c.) presente, regalo.

$t_{0}-$, presentar $\|$ regalar.

birthiay - (c.) regalos de cumpleaños.

Christmas -s, regalos de Pascuas.

$N e$ year $-s$, regalos de año nuevo, aguinaldos.

presentation copy. ejemplar con dedicatoria.

on - (c.) á presentacion.

preservation, preservacion.

preservative, preservativo.

preservatory, aparato para conservar los aliinentos.

preserve (c.) conserva alimenticia (conf.) conserva, compota.

to - , preservar, conservar (coc.) hacer conservas.

- fruits in sugar, almibarar.

- meat, curar carne.

- valie ( $\mathrm{m}$ v ) válvula de chorro ó de escape del cilindro.

TABla 8. Muestra del Diccionario tecnológico inglés-español y español-inglés de Ponce de León (1893), parte inglés-español ${ }^{14}$

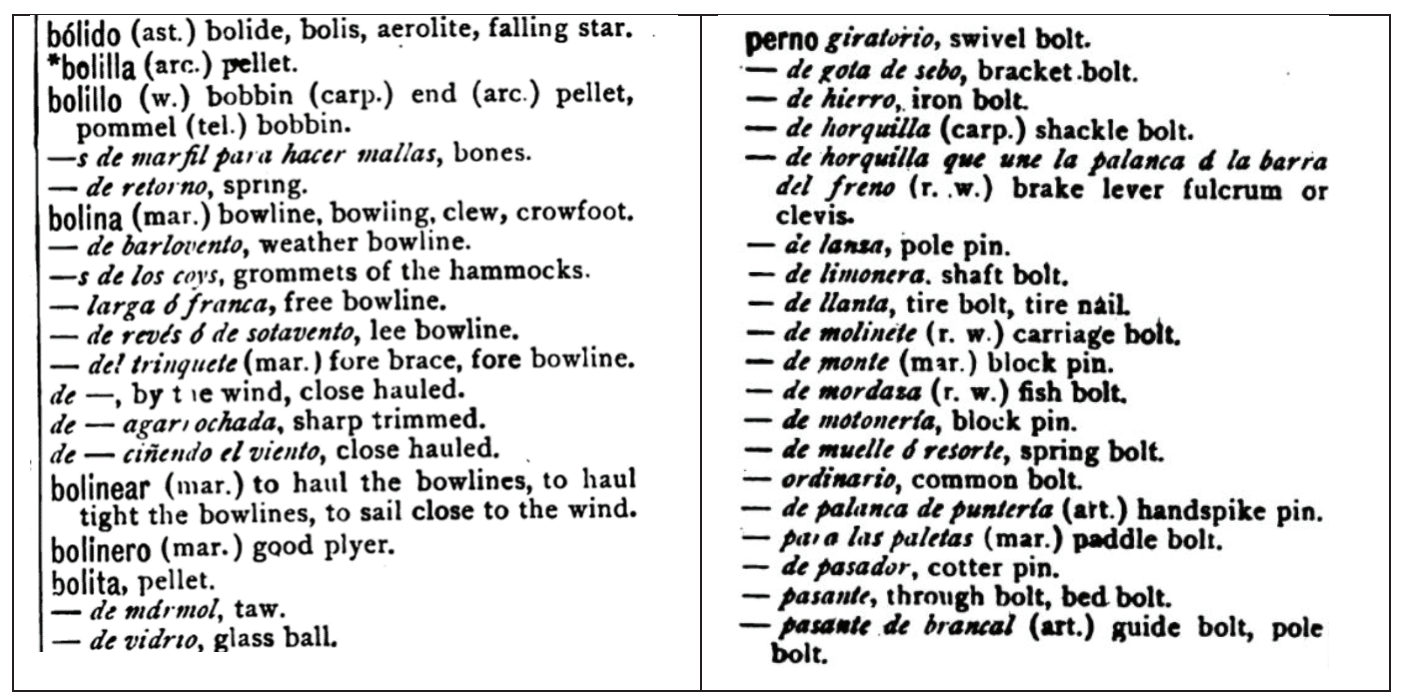

TABla 9. Muestra del Diccionario tecnológico inglés-español y español-inglés de Ponce de León (1893), parte español-inglés ${ }^{15}$

\footnotetext{
${ }^{14}$ Ponce de León: Diccionario tecnológico inglés-español y español-inglés (1893). https:// babel.hathitrust.org/cgi/pt?id=uc1.b3127112\&view=1up\&seq=7\&skin=2021.

15 Ponce de León: Diccionario tecnológico inglés-español y español-inglés (1893). https:// archive.org/details/diccionariotecn00legoog?view=theater.
} 
Son repertorios con sencillos artículos lexicográficos que tratan de facilitar la equivalencia en la otra lengua de numerosos términos especializados. Ponce de León resume muy bien los objetivos, el contenido y las dificultades que ha encontrado al realizarlo:

En vista del extraordinario desarrollo que ha alcanzado el comercio entre los países donde se habla la lengua inglesa y en los que domina la castellana, desarrollo que va tomando cada día mayor incremento, el estudio de ambos idiomas es hoy indispensable para los que en ese comercio se ocupan. Diariamente es preciso traducir cartas, pedidos, facturas, \&c., \&c., que a más de dar inmenso trabajo ocasionan pérdida de tiempo y grandes y perjudiciales errores, por falta de un diccionario como el que hoy me atrevo a presentar al público, pues no existe ninguno que dé los términos técnicos, ni aun siquiera los de su propia lengua [...]. Debo advertir que este libro no es un diccionario de definiciones, sino simplemente de equivalencias; a imitación de todos los diccionarios análogos, solo doy definiciones de aquellas palabras que por representar objetos, aparatos, instrumentos o aplicaciones nuevas, no se encuentran en ningún diccionario de la lengua castellana: de lo contrario, las proporciones del libro hubieran tenido que ser enormes y su precio lo pondría fuera del alcance de las personas que más lo necesitan. Una de las dificultades mayores con que he tenido que luchar ha sido la variedad de nombres con que a veces es conocido un mismo objeto en las diferentes provincias de España y en los diversos países de América en que se habla la lengua española. He aceptado siempre con preferencia los términos que se encuentran en alguno de los diccionarios de la lengua, pues los demás en su mayoría solo los conozco por catálogos, generalmente muy mal traducidos o por informes verbales de personas de la profesión, muchas de las cuales, por su falta de instrucción, no me prestaban suficiente garantía para considerarlas autoridad (1893: iii y iv)

\subsection{REPERTORIOS TEMÁticos}

Finalmente, es necesario mencionar en este panorama de la lexicografía española los repertorios ordenados por ámbitos designativos, las nomenclaturas, que no son sino una adaptación de las que venían componiéndose para le enseñanza de segundas lenguas desde la Edad Media (cf. Alvar Ezquerra, 2013; Ayala Castro, 1992a, 1992b y 1998; García Aranda, 2006, 2007 y 2010). La ordenación temática o por ámbitos designativos es poco original en la historia de la lexicografía: el desconocimiento del griego y del latín había obligado a la elaboración de repertorios bilingües ordenados por materias que heredaron, en mayor o menor medida, casi todas las lenguas modernas y que, con el paso del tiempo, fueron adaptándose a las nuevas necesidades 
sociales, económicas y políticas de unas sociedades que habían cambiado, convirtiéndose así en materiales útiles y muy prácticos para desenvolverse en situaciones cotidianas. Estas nomenclaturas, con variaciones mínimas, acompañaron a lo largo de los siglos a gramáticas, diccionarios, diálogos y métodos de enseñanza de lenguas y se convirtieron, por su didactismo, en una de las formas más prácticas e inmediatas de aprender el léxico de una lengua.

La lexicografía estadounidense no fue una excepción y también las utilizó para enseñar el léxico español. Una de las más elaboradas fue la que se incluye en la versión adaptada al español del método Dufief de enseñanza de inglés (Nature displayed in her mode of teaching languages to man, Filadelfia, Thomas L. Plowman, 1804) bajo el título La naturaleza descubierta en su modo de enseñar las lenguas a los hombres (1811, T. y G. Palmer, Filadelfia), que iba firmada por los profesores de Gramática general Manuel de Torres y Louis Hargous; unos años más tarde, en 1825, Mariano Velázquez de la Cadena colabora en la nueva adaptación de la obra (cfr. García Aranda, 2016).

El método Dufief, surgido a partir de las dificultades que tuvo el propio autor al aprender inglés (no fue el único autor de una metodología que no era filólogo o pedagogo especializado, un intelectual con un gran bagaje cultural y grandes dotes de observación), se reeditó en numerosas ocasiones y su "naturaleza vanguardista marcó la pauta para manuales posteriores en la línea del método natural" (Gallardo, 2003, 6.2). Dufief propone un método basado en el aprendizaje de la lengua materna (método natural, que no se desviara, como en la educación formal, de la naturaleza, pues en la naturaleza reside la sabiduría: seguir la naturaleza para desarrollar en el alumno los principios mismos de la razón y, junto a ellos, los principios de la organización del mundo), en el uso del idioma en aspectos de la vida cotidiana.

El método Dufief incluye varios vocabularios, conversaciones y diálogos, gramática, modelos de correspondencia y extractos de textos literarios, contenido que no sorprende en la historia de la enseñanza de segundas lenguas (Sánchez Pérez, 1992) pero que con Dufief sufre una importante adaptación 
(se autodefinen como vanguardistas, originales e innovadores, pero se sirven de ideas pedagógicas y lingüísticas existentes; el eclecticismo es su rasgo definidor).

El primer volumen de La naturaleza descubierta está dedicado exclusivamente al vocabulario, parte más importante para el principiante "pues le facilita adquirir un completo conocimiento de la fraseología de la lengua inglesa" (1811, vi), ya que "a language is a collection of the modes of expression made use of by the individuals of a nation in order to express their thoughts" (1811, 1), y que Dufief divide en tres apartados: el primero "comprehende los nombres de aquellos objetos que concurren freqüentemente en la conversación” y en cuya presentación "se ha procurado seguir, en quanto ha sido posible, el orden en que nos los presentan nuestras necesidades en la sociedad", esto es, por ámbitos designativos; el segundo "contiene las varias especies de Números, los principales Adjetivos y una colección de Nombres Abstractos" y el tercero "es una serie de las voces más importantes, a saber, aquellas que forman el enlace y complemento del sentido entre las partes de la oración, y se conocen en la gramática por la denominación de Artículos, Pronombres, Preposiciones, Adverbios, Conjunciones e Interjecciones" (1811, vii).

La novedad que incluye Dufief, poco explotada hasta el momento, es la de adaptar "una frase familiar de aquellas que comúnmente se usan con la misma voz. De este modo la frase familiar hace más patente la voz fundamental en cada vocabulario, de la misma suerte que un quadro excelente da más realce a la pintura que contiene" (1811, vii); así, por ejemplo, "table-mesa" va acompañada de "the table is too small for twenty persons-la mesa es demasiado pequeña para veinte personas", "brunette-morena" de "some like brunettes and others fair women-unos quieren a las morenas y otros prefieren a las rubias", "collier-carbonero" de "at the sight of so many dirty colliers I thought I was in the infernal regions-me creí en el infierno quando me vi en medio de tantos y tan sucios carboneros", "dog-perro" de "he has taught his dog to perform tricks, to walk on two feet, to dance, to fetch and carry, to leap at the king's name and at the queen's, and to counterfeit death-ha enseñado a 
su perro a hacer varias vueltas, a andar en dos pies, a balar, a traer, a saltar por el rey y la reyna, y a hacer el mortecino" y así todas las voces recogidas.

Estos ejemplos o modelos de uso son muy útiles para mostrar los rasgos morfológicos, sintácticos y ortográficos de una lengua (conjugaciones, concordancias, género, número, normas de acentuación ...), para completar la información semántica -pues la brevedad de los artículos lexicográficos impide ofrecer datos sobre significados, acepciones o sentidos-, para insertar algunos consejos (comma-coma: "quando lea ponga vmd. atención a las comas"; colon-dos puntos: "deténgase vmd. un poco más; hay dos puntos"; hyphen-rasguito: "estas son dos palabras compuestas que es necesario liar con un rasguito") y para introducir detalles sobre los usos, costumbres e ideología característicos de la centuria, lo que resultaba muy útil para el estudiante, pues muchas veces no disponía de otros medios para conocer esa información.

La nomenclatura o "nombres de objetos que concurren frecuentemente en la conversación" está compuesta por más de dos mil quinientas cincuenta voces inglesas y sus correspondientes equivalentes, y abarca el léxico básico y usual de la lengua, lo que es habitual en la mayoría de las nomenclaturas del español.

Dufief agrupa las voces (sustantivos y adjetivos) en cuatro: primero comida y vestido, después la ciudad, luego los animales y las plantas y finalmente el universo, organización del mundo que es habitual en las nomenclaturas desde el siglo XVI, momento en que se pasa de un orden teocentrista (Dios y la religión solían ser los primeros epígrafes) a uno antropocentrista (en que el hombre se sitúa en el centro de la descripción del mundo).

A lo largo de la centuria otras muchas nomenclaturas o repertorios ordenados temáticamente vieron la luz en Estados Unidos: Mariano Cubí y Soler ( A concise and useful Vocabulary of the most necessary Words, Baltimore, Jun Fielding Lucas, 1822), Edward Barry (The elements of Spanish and English conversation: with new, familiar, and easy dialogues, designed particularly 
for the use of schools, Filadelfia, H. C. Carey and I. Lea, 1822) o F. F. Mortiz Foerster (A new practical and easy method of learning the Spanish language, after the system of F. Abn [...]. First course, Nueva York, D. Appleton and Company, 1883) también utilizaron una ordenación no alfabética para presentar el léxico en sus respectivas obras.

Y también aparece en el vocabulario que se encuentra dentro de ¿Habla Vm. español? or Do you Speak English? A pocket companion for beginners who wish to acquire the facility of expressing themselves fluently on every-day topics in a short, easy and practical way. With hints on Spanish pronunciation (Nueva York, Henry Holt y Frederick Leypoldt, 1868), un manualito anónimo que tenía como objetivo reunir una serie de contenidos para aprender español de forma "short, easy and practical" (portada). Sin preliminares ni autoría conocida, la obra debió surgir por las necesidades que imponían las nuevas circunstancias políticas y sociales, en donde los viajes y las transacciones entre Estados Unidos y España eran frecuentes (García Aranda, 2018).

En esta ocasión, el léxico se agrupa bajo las estaciones, los días de la semana, los meses del año, los números cardinales, los números ordinales, del tiempo (un siglo, una semana, el crepúsculo, el romper del día, la tarde, antes de ayer, pasado mañana, el medio), comidas y bebidas (el chocolate, el té, el cacao, el pan, molletes, carne, cocido, tocino, jamón, sopa, pastel, torta, gallina, perdiz, caballa, pimienta, habas, vinagre, vino, ginebra) y vestidos (sombrero, corbata, bata, tirantes, calzoncillos, ligas, botas, escarpines, bastón, botones, ojales, zarcillos, abanico, hilo, peine). Dados los ámbitos designativos en que se distribuye el vocabulario, es fácil suponer que la mayoría de artículos lexicográficos son sinonímicos simples formados por sustantivos (véase Tabla 10).

Las nomenclaturas, de ser un instrumento de acercamiento a una lengua muerta, pasaron a reflejar las necesidades sociales, económicas y políticas de una sociedad que había cambiado, convirtiéndose así en materiales útiles y muy prácticos para desenvolverse en situaciones cotidianas. 


\begin{tabular}{|ll|ll|}
\hline El almuerzo & Breakfast & Un vestido & A suit of clothes \\
Las once & Lunch & Un sombrero & A hat \\
La comida & Dinner & Un cuello & A collar \\
El té & Tea, or the tea & Una corbata & A cravat \\
La cena & Supper & Un corbatin & A stock \\
El té & Tea & Una bata & A night-shirt \\
El café & Coffee & Una camisa & A shirt, a chemise \\
El chocolate & Chocolate & Una casaca, ó un fra- A coat \\
El cacao & Cocoa & que & Sleeves \\
La leche & Milk & Mangas & \\
La crema, ó nata & Cream & Bolsillos, ó faltrique- Pockets \\
El pan & Bread & ras & \\
La manteca, mante- & Butter & & \\
-- quilla & - & & \\
\hline
\end{tabular}

TABla 10. Muestra de ¿Habla Vm. español? (1868)

\section{CONCLUSIONES}

Este somero y general panorama de la lexicografía española en Estados Unidos muestra la actividad diccionarística que se desarrolló durante el siglo XIX al otro lado del Atlántico. Se compusieron diccionarios alfabéticos, nomenclaturas, repertorios para viajeros, para estudiantes, para comerciantes y para científicos, diccionarios generales, diccionarios de pronunciación, repertorios bilingües y multilingües...

Aunque estas páginas ponen de manifiesto que es necesaria una investigación rigurosa y detallada sobre cada uno de los diccionarios, vocabularios y glosarios publicados en ese país con el objetivo de describirlos y catalogarlos, este primer acercamiento sirve para conocer el trabajo realizado por profesores, lexicógrafos y lingüistas en una época y un área geográfica todavía poco conocidas.

\section{BIBLIOGRAFIA}

Alvar Ezquerra, M. (1992). Tradición en los diccionarios del español. Revista Española de Lingüística, 22/1, pp. 1-23.

Alvar Ezquerra, M. (2002). De antiguos y nuevos diccionarios del español. Arco/Libros.

Alvar Ezquerra, M. (2012). Las nomenclaturas del español: siglos XV-XIX. Liceus.

${ }_{16}$ ¿Habla Vm. español? (1868). https://books.google.es/books?id=urLQ8CBCKHIC\&hl= es\&pg $=\mathrm{PA} 1{ }^{\#} \mathrm{~V}=$ onepage\&q\&f=false. 
Ayala Castro, M. C. (1992a). El concepto de nomenclatura. En M. Alvar Ezquerra (Ed.), Actas del IV Congreso Internacional Euralex'90. Biblograf, pp. 437-444.

Ayala Castro, M. C. (1992b). Nomenclatures de l'espagnol (1526-1800). Considérations générales sur la nature et la fonction des nomenclaturas. Cabiers de lexicologie, LXI, pp. 127-160.

Ayala Castro, M. C. (1998). Los otros diccionarios del español: clasificaciones metódicas del siglo XIX. En M. Alvar Ezquerra y G. Corpas Pastor (Coords.), Diccionarios, frases, palabras. Servicio de Publicaciones de la Universidad, pp. 85-100.

Biblioteca Virtual de la Filología Española (BVFE). Directorio bibliográfico de gramáticas, diccionarios, obras de ortografía, ortología, prosodia, métrica, diálogos e historia de la lengua [en línea] <www.bvfe.es> [1/09/2021].

Dorta, J., Corrales, C. y Corbella, D. (eds.) (2007). Historiografía de la lingüística en el ámbito hispánico: fundamentos epistemológicos y metodológicos. Arco/Libros.

Doyle, G. (1926). Spanish Studies in the United States. Government Printing Office.

Esparza Torres, M. Á. y Niederehe, H.-J. (2012). Bibliografía cronológica de la lingüística, la gramática y la lexicografía del español (BICRES IV). Desde el año 1801 hasta el año 1860. John Benjamins Publishing. https://doi.org/10.1075/sihols.118.

Esparza Torres, M. Á. y Niederehe, H.-J. (2015). Bibliografía cronológica de la lingüística, la gramática y la lexicografía del español (BICRES V). Desde el año 1861 hasta el año 1899. John Benjamins Publishing. https://doi.org/10.1075/sihols.124.

Gallardo Barbarroja, M. (2002). La enseñanza de lenguas extranjeras en el siglo XIX: análisis de algunos métodos publicados en Inglaterra para el aprendizaje de la lengua española. Boletín de la Sociedad Española de Historiografía Lingüistica, 3, pp. 89-106.

Gallardo Barbarroja, M. (2003). Introducción y desarrollo del español en el sistema universitario inglés durante el siglo XIX. Estudios de Lingüística del Español (ELiEs), 20.

García Aranda, M. á. (2006). La enseñanza del léxico latino en el Renacimiento: Nebrija, su "Lexicon seu parvum vocabularium" y las nomenclaturas del español. Servicio de Publicaciones.

García Aranda, M. ${ }^{a}$ Á. (2007). La clasificación conceptual del léxico en repertorios españoles de los Siglos XVI y XVII. Revista de Lexicografía, 7, pp. 69-76.

García Aranda, M. ${ }^{a}$ Á. (2010). Nomenclaturas decimonónicas del español. Boletín de Lingüistica, 22, pp. 5-28.

García Aranda, M. ${ }^{a}$ Á. (2012). Lexicografía. En A. Zamorano (Coord. y Ed.), Reflexión lingüística y lengua en la España del XIX: marcos, panoramas y nuevas aportaciones. Lincom, pp. 116-138.

García Aranda, M. á Á. (2016). La enseñanza del léxico en el método Dufief: La naturaleza descubierta en su modo de enseñar las lenguas a los hombres (Filadelfia, 1811). Verba: Anuario galego de filoloxia, 43, pp. 329-359. https://doi.org/10.15304/verba.43.2536.

García Aranda, M. ${ }^{a}$ Á. (2018). El español es una lengua bastante fácil, si solo se trata de hablarlo un poco para hacerse uno entender: métodos de bolsillo para aprender 
español en Nueva York (1868). RAHL: Revista argentina de historiografía lingüistica, 10, pp. 107-124.

Garriga, C. y Gallego, R. (2008). Velázquez de la Cadena y la lexicografía bilingüe inglés/ español. En J. A. DeCesaris y E. Bernal (Coords.), Proceedings of the XIII EURALEX International Congress (Barcelona, 15-19 July 2008). Institut Universitari de Lingüística Aplicada, Universitat Pompeu Fabra, pp. 1105-1114.

Hartmann, R. R. K. (2003). Lexicography: Lexicography, metalexicography and reference science. Routledge.

Leavitt, S. E. (1961). The teaching of Spanish in the United States. Hispania, 44, pp. 591-625. https://doi.org/10.2307/336603.

Martín-Gamero, S. (1961). La enseñanza del inglés en España (desde la Edad Media hasta el siglo XIX). Gredos.

Medina Guerra, A. M. ${ }^{a}$ (coord.) (2003). Lexicografía española. Ariel.

Mühlschlegel, U. (2011). De paisajes y palabras: Josep Baretti, viajero y lexicógrafo. En R. Musser (Ed.), El viaje y la percepción del otro: viajeros por la Península Ibérica y sus descripciones (siglos XVIII y XIX). Iberoamericana-Vervuert, pp. 99-107.

Nichols, M. W. (1945). The History of Spanish and Portuguese teaching in the United States. En G. Doyle (Ed.), A Handbook on the teaching of Spanish and Portuguese with special reference to Latin America. D. C. Heath, pp. 99-146.

Piccioni, L. (1899). Studi e ricerche intorno a Giuseppe Baretti. Raffaello Giusti.

Sánchez Pérez, A. (1992). Historia de la ensenanza del espanol como lengua extranjera. SGEL.

Spell, J. R. (1927). Spanish teaching in the United States. Hispania, 10, pp. 141-159. https:// doi.org/10.2307/331235.

Steiner, R. (1970). Two Centuries of Spanish and English Bilingual Lexicography (1590-1800). Mouton. https://doi.org/10.1515/9783110807363.

Vilar García, M. (1996). La prensa en los orígenes de la enseñanza del español en los Estados Unidos (1823-1833). Servicio de publicaciones de la Universidad de Murcia.

Vilar García, M. (2008). El español, segunda lengua en los Estados Unidos: de su enseñanza como idioma extranjero en Norteamérica al bilingüismo. EDITUM. 